\title{
Parameter Synthesis for Probabilistic Timed Automata Using Stochastic Game Abstractions
}

\author{
Aleksandra Jovanović and Marta Kwiatkowska \\ Department of Computer Science, University of Oxford, Oxford, OX1 3QD, UK
}

\begin{abstract}
We propose a method to synthesise optimal values of timing parameters for probabilistic timed automata, in the sense that the probability of reaching some set of states is either maximised or minimised. Our first algorithm, based on forward exploration of the symbolic states, can only guarantee parameter values that correspond to upper (resp. lower) bounds on maximum (resp. minimum) reachability probability. To ensure precise reachability probabilities, we adapt the game-based abstraction refinement method. In the parametric setting, our method is able to determine all the possible maximum (or minimum) reachability probabilities that arise for different values of timing parameters, and yields optimal valuations represented as a set of symbolic constraints between parameters.
\end{abstract}

\section{Introduction}

Stochastic aspect is very important for modelling numerous classes of systems, such as communication and security protocols, due to component failures, unreliable channels or randomisation. The correctness of such systems can be guaranteed only with some probability. Many of them also operate under timing constraints. In such cases, the probability of a property being true depends on those timing aspects in the system: for example, increasing a certain delay might increase the maximum or minimum probability of reaching an error state.

Automatic synthesis of timing constraints to ensure the satisfaction of a given property has received a lot of attention lately. Its aim is to overcome the disadvantage of model checking, which requires complete knowledge of the system. This is often difficult to obtain, especially in the early design stages, when the whole environment is not yet known. The use of parameters instead of concrete values gives more freedom to the designers. A parametric timed model can specify that a transition is enabled for $a$ time units, where $a$ is a parameter. The goal is then to automatically synthesize the values of model's parameters such that the specification is guaranteed. Parameterisation, however, makes verification more difficult, as most problems become undecidable.

In this paper, we are dealing with the synthesis of timing parameters for probabilistic real-time systems modelled as probabilistic timed automata (PTA) [18]. PTA have been introduced as an extension of timed automata (TA) [1] for modelling and analysing systems which exhibit real-time, nondeterministic and 
probabilistic behaviour. They are finite-state automata extended with clocks, real-valued variables which increase at the same, constant rate. A fundamental property of PTA is the maximum/minimum probability of reaching a certain set of states in the model (i.e. the reachability probabilities). These probabilities allow one to express a broad range of properties, from quality of service to reliability, for example, deadline properties: "the maximum probability of an airbag failing to deploy within 0.02 seconds". PTA have been successfully used to analyse protocols such as FireWire, Bluetooth, IEEE 802.11, etc. These are embedded in a networked environment and their properties are almost always expressed parametrically, as concrete values make sense only when the network environment is known. It is thus desirable to provide a tool to automatically derive the constraints on parameters for probabilistic systems, so that their correctness is ensured with optimal probability.

Contributions We propose an algorithm for parameter synthesis for PTA based on the symbolic state-space exploration. As the forward approach gives only upper (resp. lower) bounds on max. (resp. min.) reachability probability, we adapt the game-based abstraction refinement method. This method has been introduced in [13] for Markov decision processes, and extended in [15] for PTA, for the computation of exact $\mathrm{max} / \mathrm{min}$ reachability probabilities. As we consider parametric models, these probabilities are not unique and depend on particular parameter valuations. Our algorithm allows us to choose the valuations for which these probabilities are either maximised or minimised, and to synthesise them as a finite set of symbolic constraints on parameters. To the best of our knowledge, this is the first paper dealing with optimal timing parameter synthesis for probabilistic timed automata. A full version of this paper is available as [10].

Related work An orthogonal line of work on parameter synthesis for untimed probabilistic models is that of [7], where the authors consider Markov chains and transition probabilities as parameters. Regarding timed systems, parametric timed automata have been introduced in [2] as a means to specify parametric timing constraints. The reachability-emptiness problem, which asks whether there exists a parameter valuation such that the automaton has an accepting run, is undecidable. Subsequent research has thus concentrated on finding subclasses for which certain problems would be decidable by restricting the use of parameters [9] or by restricting the parameter domain [11]. In [6], the authors consider fully deterministic networks of timed automata with priorities and parametric guards, and extended MTL with counting formulas. They develop an algorithm based on constraint solving and Monte Carlo sampling to synthesise timing delays. There is little work, however, on timing parameter synthesis for probabilistic real-time systems. In [8], a technique is proposed to approximate parametric rate values for continuous-time Markov chains for bounded reachability probabilities. In [3], the authors apply their Inverse method for parameter synthesis for TA to PTA. The method starts from reference parameter values of a TA, and derives the constraints on parameters such that the obtained models are time-abstract equivalent. Time-abstract equivalence preserves untimed properties, and thus the parameter values derived on the non-probabilistic version of 
the model preserve reachability probabilities. Termination is not guaranteed and the derived constraints are not weakest in general. In [4], the authors consider a fully deterministic parametric model, where the remaining time in a node is unique and given as a parameter, and provide a method to compute the expected time to reach some node as a function of model's parameters.

\section{Preliminaries}

A discrete probability distribution over a set $S$ is a function $\mu: S \mapsto[0,1]$, such that $\sum_{s \in S} \mu(s)=1$ and the set $\{s \mid s \in S \wedge \mu(s)>0\}$ is finite. By $\operatorname{Dist}(S)$ we denote the set of such distributions. $\mu_{p}$ is a point distribution if $\mu_{p}(s)=1$ for some $s \in S$. We now define Markov decision processes, a formalism for modelling systems which exhibit both nondeterministic and probabilistic behaviour.

Definition 1 (Markov decision processes). An $M D P$ is a tuple $\mathcal{M}=\left(S, s_{0}, \Sigma\right.$, Steps $\left._{\mathcal{M}}\right)$, where $S$ is a set of states, $s_{0}$ is a set of initial states, $\Sigma$ is a set of actions and $\operatorname{Steps}_{\mathcal{M}}: S \times \Sigma \mapsto \operatorname{Dist}(S)$ is a probabilistic transition function.

A transition in $\mathcal{M}$ from state $s$ is first made by nondeterministically selecting an action $\delta \in \Sigma$ and then the successor state $s^{\prime}$ is chosen randomly according to the probability distribution $\operatorname{Steps}_{\mathcal{M}}(s, \delta)$. A path is a sequence of such transitions and represents a particular resolution of both nondeterminism and probability. A state $s$ is reachable in $\mathcal{M}$ if there exists a path from the initial state of $\mathcal{M}$ to $s$. A strategy $\mathcal{A}$ is a function from finite paths to distributions which resolves nondeterminism in an MDP. For a fixed strategy $\mathcal{A}$, the behaviour of an MDP is purely probabilistic, and we can define the probability $p_{s}^{\mathcal{A}}(F)$ of reaching a target set $F \subseteq S$ from $s$ under $\mathcal{A}$. By quantifying over all strategies in $\mathcal{M}$, we can define the minimum and maximum probability of reaching $F$ :

$$
p_{\mathcal{M}}^{\min }(F)=\inf _{s \in s_{0}} \inf _{\mathcal{A}} p_{s}^{\mathcal{A}}(F) \text { and } p_{\mathcal{M}}^{\max }(F)=\sup _{s \in s_{0}} \sup _{\mathcal{A}} p_{s}^{\mathcal{A}}(F)
$$

These values can be computed efficiently together with the corresponding strategies using, e.g., value iteration, which approximates the probability value.

Stochastic 2-player games [5] are turn-based games involving two players and probability. They generalise MDPs by allowing two types of nondeterministic choice, each controlled by a separate player.

Definition 2 (Stochastic games). A stochastic game is a tuple $\mathcal{G}=\left(S,\left(S_{1}, S_{2}\right)\right.$, $s_{0}, \Sigma$, Steps $\left._{\mathcal{G}}\right)$, where $S$ is a set of states partitioned into sets $S_{1}$ and $S_{2}, s_{0}$ is a set of initial states, $\Sigma$ is a set of actions and $\operatorname{Steps}_{\mathcal{G}}: S_{1} \times \Sigma \times S_{2} \mapsto 2^{\text {Dist }(S)}$ is a probabilistic transition function.

$S_{1}$ and $S_{2}$ represent the sets of states controlled by player 1 and player 2, respectively. The behaviour of a game is as follows: first player 1, in state $s \in S_{1}$, selects an available action $\delta \in \Sigma$, which takes the game into a state $s^{\prime} \in S_{2}$. Player 2 then selects a probability distribution $\mu$ from the set $\operatorname{Steps}_{\mathcal{G}}\left(s, \delta, s^{\prime}\right)$. Finally, the successor state $s^{\prime \prime}$ is chosen according to $\mu$. A resolution of nondeterminism in $\mathcal{G}$ is a pair of strategies $\sigma_{1}, \sigma_{2}$ for player 1 and player 2 , respectively, under which we can define the probability $p_{s}^{\sigma_{1}, \sigma_{2}}(F)$ of reaching a subset $F \subseteq S$ from state $s$. 
Clocks and parameters. Let $\mathbb{R}, \mathbb{R}_{\geqslant 0}$ and $\mathbb{Z}$ be the sets of reals, non-negative reals and integers, respectively. Let $X$ be a finite set. A linear expression on $X$ is an expression of the form $\lambda:=k|k \cdot x| \lambda+\lambda$, where $k \in \mathbb{Z}$ and $x \in X$.

Now let $X=\left\{x_{1}, \ldots, x_{n}\right\}$ be a finite set of clock variables. A clock valuation $u: X \mapsto \mathbb{R}_{\geqslant 0}$ is a function assigning a non-negative real number to each $x \in X$. Let $\mathbf{0}$ be a valuation that assigns 0 to all clocks in $X$. For any $R \subseteq X$ and any valuation $u$ on $X$, we write $u[R]$ for the valuation on $X$ such that $u[R](x)=0$ if $x \in R$ and $u[R](x)=u(x)$ otherwise. For $t \geqslant 0, u+t$ denotes the valuation which assigns $(u+t)(x)=u(x)+t$ to all $x \in X$. Let $P=\left\{p_{1}, \ldots, p_{m}\right\}$ be a finite set of parameters. A (linear parametric) constraint on $X \cup P$ is an expression of the form $\gamma:=x_{i} \sim c\left|x_{i}-x_{j} \sim c\right| \gamma \wedge \gamma$ where $1 \leqslant i \neq j \leqslant n, x_{i}, x_{j} \in X$, $\sim \in\{<, \leqslant\}$ and $c$ is a linear expression on $P$. By $\mathcal{C}(X, P)$ we denote the set of such parametric constraints and by $\mathcal{C}^{\prime}(X, P)$ we denote the set of (non-diagonal) constraints of the form: $\gamma^{\prime}:=x_{i} \sim c \mid \gamma^{\prime} \wedge \gamma^{\prime}$. For any valuation $v$ on $P$ and any linear constraint $\gamma$ on $X \cup P, v(\gamma)$ is the linear constraint on $X$ obtained by replacing each parameter $p \in P$ by the (concrete) value $v(p)$. Given some arbitrary order on $X \cup P$, a valuation can be viewed as a real-valued vector of size $|X \cup P|$. The set of valuations satisfying some linear constraints is then a convex polyhedron of $\mathbb{R}^{|X \cup P|}$. A zone is a polyhedron defined only by conjunctions of the constraints of the form $x-y \sim c$ or $x \sim c$ with $x, y \in X, c \in \mathbb{Z}$ and $\sim \in\{<, \leqslant\}$. If $v$ is a valuation on both clocks and parameters $X \cup P$ (as $v$ is used throughout the paper, unless specified otherwise) then by $v_{\mid P}$ (resp. $v_{\mid X}$ ) we denote the projection of $v$ onto $P$ (resp. $X$ ). We now give a formal definition of Parametric Probabilistic Timed Automata (PPTA), which are PTA extended with timing parameters.

Definition 3 (PPTA). A PPTA is a tuple $\mathcal{P}=\left(L, l_{0}, X, P, \Sigma\right.$, prob, Inv) where: $L$ is a finite set of locations; $l_{0} \in L$ is the initial location; $X$ is a finite set of clocks; $P$ is a finite set of parameters; $\Sigma$ is a finite set of actions; prob : $L \times \Sigma \times \mathcal{C}(X, P) \mapsto \operatorname{Dist}\left(2^{X} \times L\right)$ is a probabilistic transition function; and Inv : $L \mapsto \mathcal{C}^{\prime}(X, P)$ is a function that assigns an invariant to each location.

For any rational valuation $v$ on $P$, the structure $v(\mathcal{P})$ obtained from $\mathcal{P}$ by replacing every constraint $\gamma$ by $v(\gamma)$ is a PTA. The behaviour of a PPTA $\mathcal{P}$ is described by the behaviour of all PTA $v(\mathcal{P})$ obtained by considering all possible parameter valuations. A (concrete) state of $v(\mathcal{P})$ is a pair $(l, u) \in L \times \mathbb{R}_{\geqslant 0}^{X}$ such that the clock valuation $u$ satisfies the invariant (notation $u \models v(\operatorname{lnv}(l))$ ). A transition in the semantics of $v(\mathcal{P})$ is a timed-action pair $(t, \delta)$. In each state certain amount of time $t \in \mathbb{R}_{\geqslant 0}$ can elapse, as long as $u+t \models v(\operatorname{lnv}(l))$. Time elapse is followed by the choice of an action $\delta \in \Sigma$, for which the set of clocks $R$ to reset and successor locations $l^{\prime}$ are selected randomly according to the probability distribution $\operatorname{prob}(l, \delta, \gamma)$. The action $\delta$ can only be taken once its constraint $v(\gamma)$ (called guard) is satisfied by the current clock valuation. Each element $\left(R, l^{\prime}\right) \in 2^{X} \times L$, such that $\operatorname{prob}(l, \delta, \gamma)\left(R, l^{\prime}\right)>0$, is called an edge and the set of all such edges, denoted edges $(l, \delta, \gamma)$, is assumed to be an ordered list $\left\langle e_{1}, \ldots, e_{n}\right\rangle$. We now formally define the semantics of a PPTA under a parameter valuation $v$. 
Definition 4 (Semantics of a PPTA). Let $\mathcal{P}=\left(L, l_{0}, X, P, \Sigma\right.$, prob, Inv) be a PPTA and $v$ be a $\mathbb{R}$-valuation on $P(v: P \mapsto \mathbb{R})$. The semantics of $v(\mathcal{P})$ is given by the infinite-state $M D P \mathcal{M}_{v(\mathcal{P})}=\left(Q, q_{0}, \mathbb{R}_{\geqslant 0} \times \Sigma\right.$, Steps $\left.\mathcal{M}_{\mathcal{M}_{(\mathcal{P})}}\right)$ where:

- $Q=\left\{(l, u) \in L \times X \mapsto \mathbb{R}_{\geqslant 0} \mid u \models v(\operatorname{Inv}(l))\right\}, q_{0}=\left(l_{0}, \mathbf{0}\right)$

- Steps $\mathcal{M}_{v(\mathcal{P})}((l, u),(t, \delta))=\mu$ iff $\exists\left(R, l^{\prime}\right) \in \operatorname{edges}(l, \delta, \gamma)$ such that $u+t \models$ $v(\gamma) \wedge u+t^{\prime} \models \operatorname{Inv}(l)$ for all $0 \leqslant t^{\prime} \leqslant t$, and for any $\left(l^{\prime}, u^{\prime}\right) \in Q$ :

$$
\mu\left(l^{\prime}, u^{\prime}\right)=\sum\left\{\left|\operatorname{prob}(l, \delta, \gamma)\left(R, l^{\prime}\right)\right| R \in 2^{X} \wedge u^{\prime}=(u+t)[R] \mid\right\}
$$

Note that the definition of $\mu$ involves summation over the cases in which multiple clock resets result in the same target state $\left(l^{\prime}, u^{\prime}\right)$, expressed as a multiset, since some of the probabilities might be the same.

We study the optimal timing parameter synthesis problem for PPTA, i.e., automatically finding values of parameters such that the probability (either maximum or minimum) of reaching a certain set of locations is optimised. For example, in the case of property "the maximum probability of an airbag failing to deploy", we would want to choose the timing parameters that minimise this probability value. On the other hand, we would want to maximise "the maximum probability that the protocol successfully terminates".

\section{Synthesis with Forward Reachability}

A naive approach to parameter synthesis for PTA is to restrict parameter values to bounded intervals of integers (or rationals that can be scaled to integers) and perform verification for each such (non-parametric) model using a probabilistic model checker, e.g. PRISM [16]. However, this approach is shown to be inefficient for (non-probabilistic) TA compared to symbolic techniques, especially when the sets of possible parameter values are large [11]. This is why we aim to formulate a symbolic algorithm for deriving constraints on parameters that ensure the optimisation of some reachability probability in the model. For the symbolic exploration of the state-space, we use the notion of parametric symbolic state and forward symbolic operations on valuation sets given below, defined in [11].

Definition 5 (Parametric symbolic state). A (parametric) symbolic state of a PPTA $\mathcal{P}$, with set of clocks $X$ and set of parameters $P$, is a pair $S=(l, \zeta)$ where $l$ is a location of $\mathcal{P}$ and $\zeta$ is a set of valuations $v$ on $X \cup P$.

- future (time successors): $\zeta^{\nearrow}=\left\{v^{\prime} \mid v \in \zeta \wedge v^{\prime}(x)=v(x)+d, d \geqslant 0\right.$ if $x \in$ $X ; v^{\prime}(x)=v(x)$ if $\left.x \in P\right\}$

- reset of clocks in $R \subseteq X: \zeta[R]=\{v[R] \mid v \in \zeta\}$

- successor by edge $e=\left(R, l^{\prime}\right)$ in the distribution $\operatorname{prob}(l, \delta, \gamma)$ : $\operatorname{Succ}((l, \zeta), e)=$ $\left(l^{\prime},(\zeta \cap \gamma)[R]^{\nearrow} \cap \operatorname{Inv}\left(l^{\prime}\right)\right)$

- initial symbolic state: Init $(\mathcal{P})=\left(l_{0},\left\{v \in \mathbb{R}^{X \cup P} \mid v_{\mid X} \in\left\{\mathbf{0}_{X}\right\}^{\nearrow} \wedge v\left(\operatorname{Inv}\left(l_{0}\right)\right)\right\}\right)$.

The sets of valuations of all reachable symbolic states of a PPTA are convex polyhedra [9], since the set of valuations of the initial symbolic state is a convex polyhedron and all the operations preserve convexity. 
Forward reachability exploration The forward exploration, which builds an MDP-based abstraction of a given PTA [18], is an extension of the well-known zone-based forward reachability algorithm, ubiquitous for model-checking TA. This algorithm performs the exploration of the state-space by successively computing symbolic states using Succ, starting from the initial state. For probabilistic models, on-the-fly techniques are not used, as the goal is to compute the probability of reaching a state, instead of just checking the existence of a path.

In Fig. 1 we present our extension of the forward reachability algorithm from [18] to parametric probabilistic timed automata. It takes a PPTA $\mathcal{P}$ and some subset of its locations $F$ as input, and returns the reachability graph (Sym, Trans). Sym is the set of all reachable parametric symbolic states $S$ of the model and Trans is the set of symbolic transitions. Waiting is the set of those symbolic states which have not yet been explored. As long as there are symbolic states unexplored (Waiting $\neq \varnothing$ ), successor states are computed for each possible edge using Succ operator. Each symbolic transition $T \in$ Trans is of the form $T=\left((l, \zeta), \delta,\left\langle\left(l_{1}, \zeta_{1}\right), \ldots,\left(l_{n}, \zeta_{n}\right)\right\rangle\right)$, where $n=\mid$ edges $(l, \delta, \gamma) \mid$. A symbolic transition $T$ induces probability distribution $\mu_{T}$ over symbolic states $S y m$. For any $\left(l^{\prime}, \zeta^{\prime}\right) \in \operatorname{Sym}: \mu_{T}\left(l^{\prime}, \zeta^{\prime}\right)=\sum\left\{\left|\operatorname{prob}(l, \delta, \gamma) e_{i}\right| e_{i} \in \operatorname{edges}(l, \delta, \gamma) \wedge \zeta_{i}=\zeta^{\prime} \mid\right\}$.

Using these distributions, the algorithm $\operatorname{BuILDMDP}($ Sym, Trans $)$ constructs an MDP similarly to that of [18] for PTA, which can then be analysed to compute the reachability probabilities. For PTA, and therefore for PPTA, this approach only gives upper (resp. lower) bounds on maximum (resp. minimum) reachability probability in the model. This is because the reachability graph is too coarse to preserve precise time the actions can be taken, and thus constructs an overapproximation of the possible strategies.

Let us highlight the differences between our algorithm and its non-parametric counterpart from [18]. In the non-parametric case, all the symbolic states $(l, \zeta)$ containing some location $l \in F$ are collected into a set Reached. Then, in the constructed MDP, the max. (or min.) probability of ending up in Reached is calculated. In our setting, we are interested in finding the optimal parameter valuations (that maximise or minimise some reachability probability). We thus need to keep separate those symbolic states containing different parameter valuations and calculate the $\mathrm{max} / \mathrm{min}$ reachability probability for each one. We divide the set Reached into subsets Reached ${ }_{i}$, each of which contains the symbolic states $\left(l_{i}, \zeta_{i}\right)$ with equivalent parameter values (obtained by projection onto parameters $\left.\zeta_{i \mid P}\right)$. Another difference arises when building symbolic transitions Trans. This follows from the property of TA (and therefore PTA) proven in [9], which states that weakening (resp. strengthening) the guards in any TA $\mathcal{T}$, e.g decreasing lower and increasing upper (resp. increasing lower and decreasing upper) bounds on clocks, yields an automaton whose reachable states include (resp. are subset of) those of $\mathcal{T}$. We therefore add, for any two symbolic states $\left(l_{i}, \zeta_{i}\right),\left(l_{j}, \zeta_{j}\right) \in$ Sym which satisfy $\zeta_{i \mid X} \subseteq \zeta_{j_{\mid X}} \wedge \zeta_{i \mid P} \subseteq \zeta_{j_{\mid P}} \wedge l_{i}=l_{j}$, a transition (point distribution) from $\left(l_{j}, \zeta_{j}\right)$ to $\left(l_{i}, \zeta_{i}\right)$, in order to obtain the correct probabilities in the MDP. By $\left\{\text { Reached }_{i}\right\}_{\mid P}$ in Fig. 1, we denote the parameter values contained in Reached $_{i}$. 


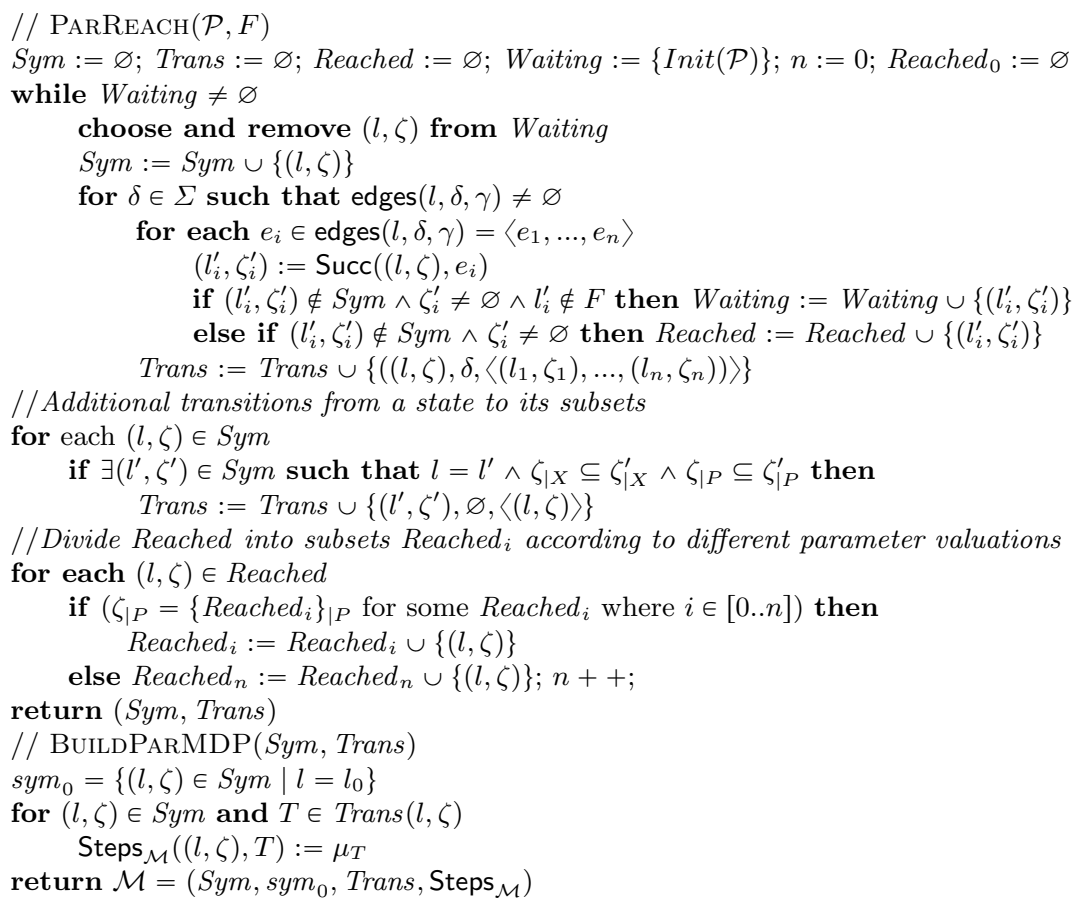

Fig. 1. Parametric forward reachability and construction of the corresponding MDP

Example 1. Let us consider a PPTA shown in Fig. 2. We are interested in the values of the parameter $b$ which maximise the probability of the medium successfully send-ing the data (reaching location $l_{2}$ ). The MDP constructed from the reachability graph is shown in Fig. 3. There are three symbolic states holding $l_{2}$ with different parameter valuations, Reached $d_{1}=\left\{\left(l_{2}, x=y \wedge b \leqslant\right.\right.$ $1)\}$, Reached $_{2}=\left\{\left(l_{2}, x=y \wedge b \leqslant 3\right)\right\}$ and Reached Re $_{3}=\left\{\left(l_{2}, x=y \wedge b \leqslant 5\right)\right\}$. Using PRISM, we calculated maximal probabilities of reaching those states in the MDP: $p^{\max }\left(\diamond\right.$ Reached $\left._{1}\right)=0.65, p^{\max }\left(\diamond\right.$ Reached $\left._{2}\right)=0.8775$, and $p^{\max }\left(\diamond\right.$ Reached $\left._{3}\right)=$ 0.957125 , where $\diamond \phi$ means that $\phi$ must hold eventually. If we want to maximise the probability of reaching $l_{2}$, it is clear that we should choose $b \leqslant 1$.

The forward reachability algorithm provides only upper (resp. lower) bound on the max. (resp. min.) reachability probability. In Example 1, this method actually gives the correct values, but consider now the automaton of Fig. 4, inspired by [18]. The probability of reaching $l_{3}$ obtained using forward approach (the resulting MDP is shown in Fig. 5) is 1, regardless of the value of $a$. By careful inspection, we observe that the max. probability is 1 only if $a=0$ (when the transition from $l_{0}$ is taken at $x=y=0$ ), and otherwise it is at most 0.5.

Theorem 1. For a PPTA $\mathcal{P}$ and a subset of its locations $F$, if $($ Sym, Trans $)=$ $\operatorname{PaRReAch}(\mathcal{P}, F)$ and $\mathcal{M}=\operatorname{BuILdMDP}($ Sym, Trans $)$, then: 


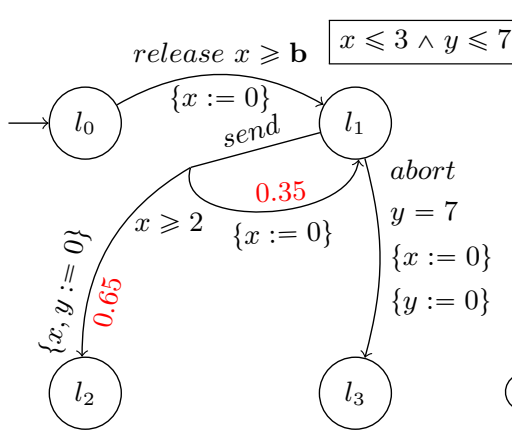

Fig. 2. PPTA

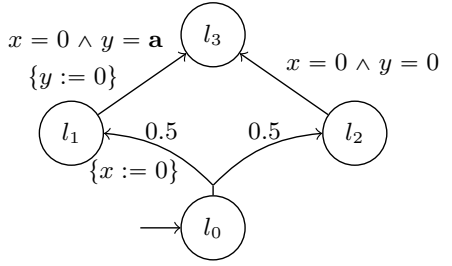

Fig. 4. PPTA

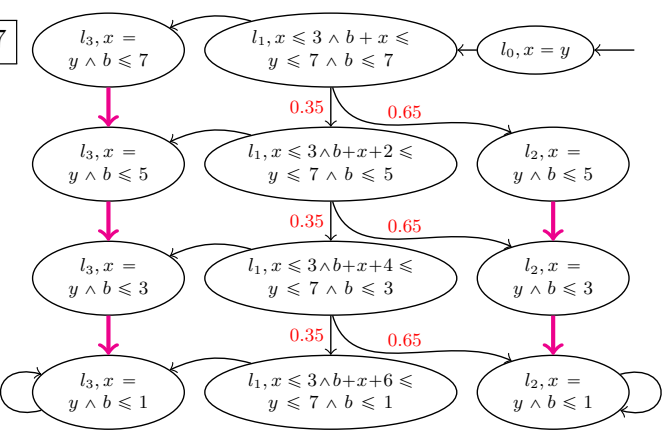

Fig. 3. MDP for PPTA of Fig. 2

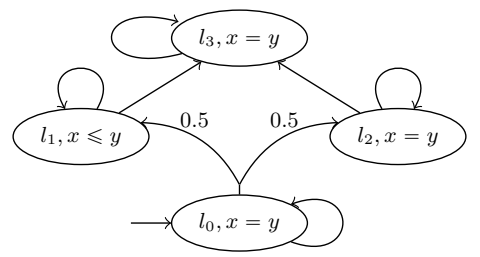

Fig. 5. MDP for PPTA of Fig. 4

- $p_{\mathcal{M}}^{\min }($ Reached $) \leqslant p_{\mathcal{P}}^{\min }(F)$ and $p_{\mathcal{M}}^{\max }($ Reached $) \geqslant p_{\mathcal{P}}^{\max }(F)$

- if $\mathcal{M}$ gives the precise reachability probabilities in $\mathcal{P}$ and if some $\left(l_{k}, \zeta_{k}\right) \in$ Reached has the optimum (max. or min.) reachability probability, among all $\left(l_{j}, \zeta_{j}\right) \in$ Reached, then $\left\{\zeta_{k \mid P} \backslash\left(\bigcup_{\forall j \neq k, l_{j} \in F} \zeta_{j \mid P}\right)\right\}$ is the solution to the optimal parameter synthesis problem.

The reachability-emptiness problem for parametric timed automata is undecidable [2], and the algorithm for forward reachability exploration for this model might not terminate $[11,9]$. Since our algorithm for PPTA can be viewed as its extension, termination cannot be guaranteed either.

To resolve the limitation of the forward approach, namely, that it can only compute bounds on the reachability probabilities, in the next section we adapt the game-based abstraction refinement method from [15] to synthesise the optimal timing parameter values for PPTA. We choose this approach as it can compute precise min. and max. probabilities and is shown to perform better then the alternative model checking technique for PTA, digital clocks [17].

\section{Synthesis with Game-based Abstraction Refinement}

In [14], stochastic two-player games are used as abstractions for MDPs. In such a game, the two players represent nondeterminism introduced by the abstraction (player 1) and nondeterminism from the original model (player 2). By quantifying over all possible strategies for players 1 and 2, we can obtain both the 
lower bound $(l b)$ and upper bound $(u b)$ on either the max. or min. reachability probability in the original MDP. If a game $\mathcal{G}$ is constructed from an MDP $\mathcal{M}$ using the approach from [14], where $F$ is a subset of states of $\mathcal{M}$, we have:

$$
p_{\mathcal{G}}^{l b, \min }(F) \leqslant p_{\mathcal{M}}^{\min }(F) \leqslant p_{\mathcal{G}}^{u b, \min }(F) \text { and } p_{\mathcal{G}}^{l b, \max }(F) \leqslant p_{\mathcal{M}}^{\max }(F) \leqslant p_{\mathcal{G}}^{u b, \max }(F) \text {. }
$$
In case of maximum probability we have: $p_{\mathcal{G}}^{l b, \max }(F) \stackrel{\text { def }}{=} \sup _{s \in s_{0}} \inf _{\sigma_{1}} \sup _{\sigma_{2}} p_{s}^{\sigma_{1}, \sigma_{2}}(F)$ and $p_{\mathcal{G}}^{u b, \max }(F) \stackrel{\text { def }}{=} \sup _{s \in s_{0}} \sup _{\sigma_{1}} \sup _{\sigma_{2}} p_{s}^{\sigma_{1}, \sigma_{2}}(F)$. Using similar techniques to value iteration for MDPs [5], these probabilities can be efficiently approximated, together with the corresponding strategy pairs which achieve them.

In [15], the concept of gamed-based abstractions is used for PTA in order to compute the maximum and minimum reachability probabilities. The method starts from the MDP obtained via forward reachability algorithm, and subsequently builds and refines the stochastic game abstraction. In this section, we generalise this method by taking into account timing parameters.

Game-based abstraction for PPTA The game-based abstraction is constructed by analysing transitions outgoing from each location in a PPTA. The transitions are divided into subsets according to the common part of the symbolic state in which they are enabled. This analysis is based on the validity operator [15]. In the non-parametric case, this operator takes the symbolic transition $T=\left((l, \zeta), \delta,\left\langle\left(l_{1}, \zeta_{1}\right), \ldots,\left(l_{n}, \zeta_{n}\right)\right\rangle\right)$ and returns false if the part of $\zeta$ from which it is possible to let time pass and then perform action $\delta$, such that taking the $i$ th edge $\left(R_{i}, l_{i}\right)$ gives some state $\left(l_{i}, v\right) \in\left(l_{i}, \zeta_{i}\right)$, is empty. Such analysis requires several backward operators, defined for the parametric domain in [12]:

- past (time predecessors): $\zeta^{\swarrow}=\left\{v^{\prime} \mid v \in \zeta \wedge v^{\prime}(x) \geqslant 0, v^{\prime}(x)+d=v(x), d \geqslant\right.$ 0 if $x \in X ; v^{\prime}(x)=v(x)$ if $\left.x \in P\right\}$

- inverse reset of clocks in set $R \subseteq X: \zeta[R]^{-1}=\left\{v^{\prime} \mid \exists v \in \zeta\right.$ s.t. $v^{\prime}(x)=$ 0 if $x \in R \wedge v^{\prime}(x)=v(x)$ otherwise $\}$

We extend the validity operator to parametric domain and replace Boolean operations with the corresponding set-theoretic operations, in order to obtain the valuations on $X \cup P$ from which it is possible to perform such a transition: $\operatorname{valid}(T)=\zeta \cap\left(\left(\gamma \cap\left(\cap_{i=1}^{n}\left(\zeta_{i}[R]^{-1}\right)\right)\right)^{\swarrow}\right)$. The transition $T$ is therefore valid if the set of valuations (polyhedron) valid $(T)$ is non-empty. The projection of these valuations onto parameters gives the corresponding values of parameters. In order to construct a stochastic game, the notion of validity is extended to sets of symbolic transitions with the same source. Again, we replace Boolean with set-theoretic operators: $\operatorname{valid}(\mathbb{T})=\left(\cap_{T \in \mathbb{T}} \operatorname{valid}(T)\right) \cap\left(\cap_{T \in \operatorname{Trans}(l, \zeta) \backslash \mathbb{T}} \neg \operatorname{valid}(T)\right)$. $\operatorname{valid}(\mathbb{T})$ defines the set of valuations $v \models \zeta$ on $X \cup P$, such that from $(l, v)$ it is possible to perform any symbolic transition $T \in \mathbb{T}$, but it is not possible to perform any other transition of $\operatorname{Trans}(l, \zeta)$. In a symbolic state $(l, \zeta)$ of a stochastic game abstraction of a PPTA, player 1 first picks a subset $\mathbb{T}$ of symbolic transitions (in other words, part of the symbolic state in which these transitions are valid), and player 2 then picks a transition $T \in \mathbb{T}$. Fig. 6 shows the algorithm for the construction of a stochastic game from a given reachability graph, which yields (by quantifying over all possible strategies for player 1 and player 2) upper and lower bounds on the $\max / \mathrm{min}$ reachability probabilities in a PPTA. 


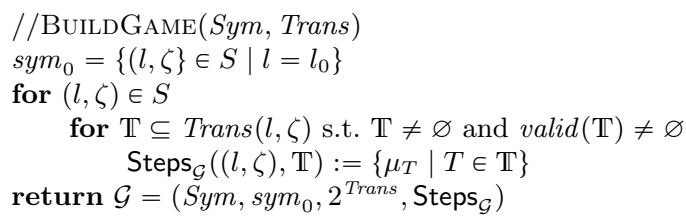

Fig. 6. Algorithm for stochastic game abstraction

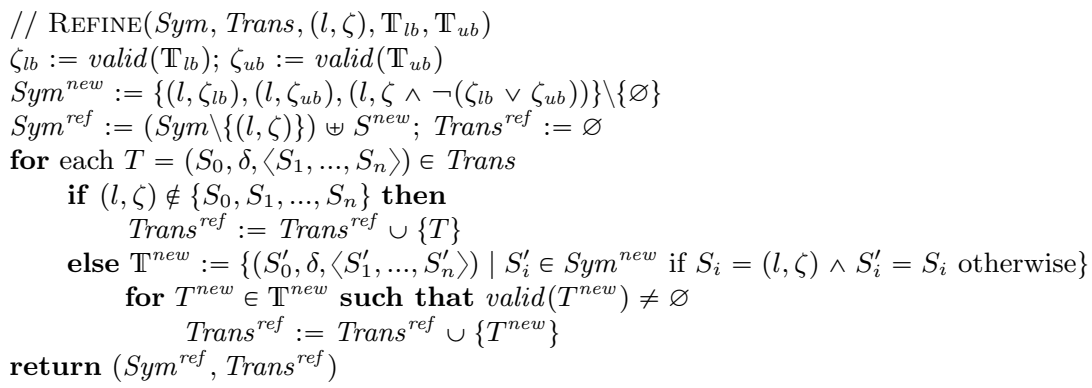

Fig. 7. Algorithm for parametric abstraction refinement

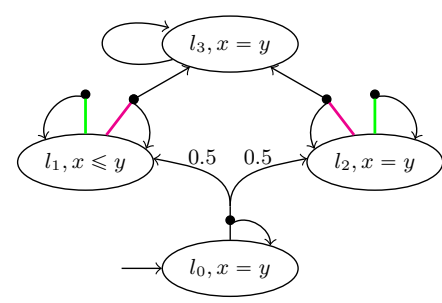

Fig. 8. Game-based abstraction

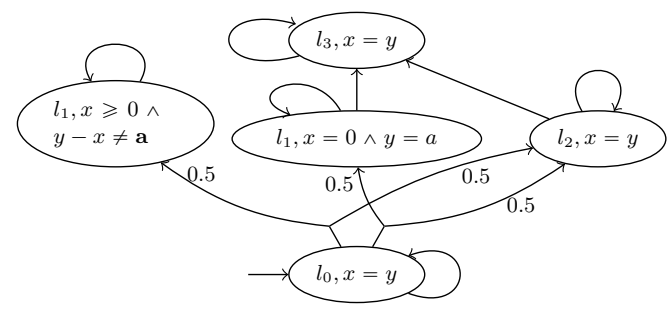

Fig. 9. Refinement of a symbolic state

Theorem 2. If (Sym, Trans $)=\operatorname{ParReach}(\mathcal{P}, F), \mathcal{G}=\operatorname{BuildGame}($ Sym, Trans $)$ and $* \in\{$ min, $\max \}$ then: $p_{\mathcal{G}}^{l b, *}($ Reached $) \leqslant p_{\mathcal{P}}^{*}(F) \leqslant p_{\mathcal{G}}^{u b, *}($ Reached $)$.

Example 2. A game constructed from the forward reachability graph of a PPTA in Fig. 2 is shown in Fig. 8. We represent player 1 states by ellipses containing symbolic states $(l, \zeta)$, and player 2 states by a black dot. In two of its states (containing $l_{1}$ and $l_{2}$ ), player 1 can choose between the part of the state where both transitions are valid and the part where only one transition is valid (a self-loop). The analysis of this game, however, gives values 0 and 1 for lower and upper bound, respectively, on the maximum probability of reaching $l_{3}$. We address this issue below by applying a method to refine the abstraction.

Parametric abstraction refinement Stochastic game abstractions might be too imprecise for reachability probabilities, as shown in Example 2. The abstraction refinement method proceeds by iteratively computing refined abstractions 
until suitable precision is obtained. The game-based abstraction refinement for MDPs from [13] uses the difference between lower and upper bounds on $\max / \mathrm{min}$ reachability probability computed thus far as the quantitative measure of precision. This method has been subsequently used in [15] for the abstraction refinement for PTA. We now explain our extension for the parametric case, which leads to parameter values corresponding to precise probabilities in the model.

After the construction and analysis of a stochastic game, the refinement algorithm, presented in Fig. 7, takes the reachability graph (Sym, Trans), splits one symbolic state per iteration and modifies symbolic transitions accordingly. The split of a symbolic state $(l, \zeta)$ is done with respect to player 1 strategy choices, $\mathbb{T}_{u b}$ and $\mathbb{T}_{l b}$, in $(l, \zeta)$, which achieve lower and upper bounds (such choices must exist in a state where these bounds differ, [14]). The symbolic state $(l, \zeta)$ is therefore split into $\left(l, \operatorname{valid}\left(\mathbb{T}_{l b}\right)\right),\left(l, \operatorname{valid}\left(\mathbb{T}_{u b}\right)\right)$, and $\left(l, \zeta \wedge \neg\left(\operatorname{valid}\left(\mathbb{T}_{l b}\right) \vee\right.\right.$ $\left.\operatorname{valid}\left(\mathbb{T}_{u b}\right)\right)$ ). By construction, both $\operatorname{valid}\left(\mathbb{T}_{l b}\right)$ and $\operatorname{valid}\left(\mathbb{T}_{u b}\right)$ are non-empty and $\operatorname{valid}\left(\mathbb{T}_{l b}\right) \neq \operatorname{valid}\left(\mathbb{T}_{u b}\right)$, and thus the split produces strict refinement. The MDP of Fig. 5, after a refinement of one symbolic state, is shown in Fig. 9.

The complete game-based abstraction refinement scheme, shown in Fig. 10, provides a means to compute the precise values for $\max / \mathrm{min}$ reachability probability, each corresponding to a particular parameter valuation. We can then choose those valuations that optimise (either maximise or minimise) these probabilities. Algorithm Synth uses function AnalyzeGame of [5] to compute bounds on $\max / \min$ probability of reaching some set of locations in a stochastic game and the corresponding strategies. The choice $\mathbb{T}_{i}$ of player 1 , in some $(l, \zeta)$, is a set of symbolic transitions $T$, and also represents the part of $\zeta$ in which these transitions are valid. To find the optimal parameter valuations, we first need to take the projection onto the parameters for each $\operatorname{valid}\left(\mathbb{T}_{i}\right)$ in the optimal strategy of player 1 (the strategy for reaching some Reached $k$ which gives the optimal probability), and take their intersection. Then, for some $\left(l_{k}, \zeta_{k}\right) \in$ Reached $_{k}$ (all of them have the equivalent $\zeta_{k \mid P}$ ), we obtain the solution as $\left\{\bigcap_{i} \operatorname{valid}\left(\mathbb{T}_{i}\right)_{\mid P} \cap\left(\zeta_{k \mid P} \backslash\left(\bigcup_{\forall j \neq k, l_{j} \in F} \zeta_{j \mid P}\right)\right)\right\}$.

Theorem 3. For a PPTA $\mathcal{P}$, a subset of its location $F$ and $* \in\{$ min, max $\}$, let $($ Sym, Trans $)=\operatorname{PARREACH}(\mathcal{P}, F)$. If $\left(\right.$ Sym $^{\text {ref }}$, Trans $\left.^{\text {ref }}\right)$ is the result returned by applying Refine to (Sym, Trans), $\mathcal{G}$ by BuildGame(Sym, Trans) and $\mathcal{G}^{\text {ref }}$ by BuILDGAme $\left(\right.$ Sym $^{\text {ref }}$, Trans $\left.{ }^{\text {ref }}\right)$ then:

- $\left(\right.$ Sym $^{\text {ref }}$, Trans $\left.^{\text {ref }}\right)$ is a reachability graph for $(\mathcal{P}, F)$;

- $p_{\mathcal{G}}^{l b, *}($ Reached $) \leqslant p_{\mathcal{G}^{\text {ref }}}^{l b, *}($ Reached $)$ and $p_{\mathcal{G}}^{u b, *}($ Reached $) \geqslant p_{\mathcal{G}^{\text {ref }}}^{u b, *}($ Reached $)$;

- If $p^{*}=p_{\mathcal{G}^{\text {ref }}}^{l b *}\left(l_{k}, \zeta_{k}\right)=p_{\mathcal{G}^{\text {ref }}}^{u b *}\left(l_{k}, \zeta_{k}\right)$, for some $\left(l_{k}, \zeta_{k}\right) \in$ Reached, is the optimum $*$ reachability probability, among all $\left(l_{j}, \zeta_{j}\right) \in$ Reached, then the solution to the optimal parameter synthesis can be extracted from the strategy $\sigma_{1}$ of Player 1 (which achieves $p^{*}$ ) and $\zeta_{k}$.

The algorithm is designed to terminate when the difference between the upper and lower bound falls below some threshold $\epsilon$ for reasons of computational efficiency. We show that this is, however, not necessary. If the initial forward reachability exploration terminates (PARREACH), then our algorithm, similarly 


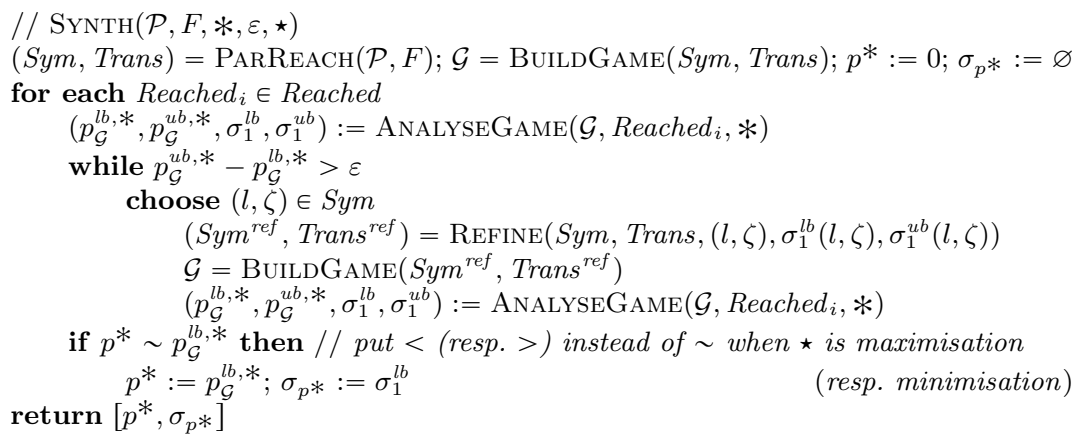

Fig. 10. Parameter synthesis using game-based abstraction refinement loop

to its non-parametric counterpart from [15], is guaranteed to terminate in a finite number of steps with a precise answer.

Theorem 4 (Termination). Let $* \in\{$ min, max $\}$. If forward reachability algorithm (PARREACH) terminates, then the algorithm for parameter synthesis SYNTH terminates after a finite number of steps and returns $p^{*}=p^{l b, *}=p^{u b, *}$.

Example 3. We return to the PPTA in Fig. 4. The final stochastic game, after two refinement iterations, contains six symbolic states. The validity of each new symbolic transition $T_{i}$, obtained in the refinement process, gives the following parameter valuations:

$-T_{1}=\left(\left(l_{0}, x=y\right), \varnothing,\left\langle\left(l_{1}, x=0 \wedge y=a\right),\left(l_{2}, x=y=0\right)\right\rangle\right) \neq \varnothing$ if $a=0$

$-T_{2}=\left(\left(l_{0}, x=y\right), \varnothing,\left\langle\left(l_{1}, x=0 \wedge y=a\right),\left(l_{2}, x=y>0\right)\right\rangle\right) \neq \varnothing$ if $a \neq 0$

$-T_{3}=\left(\left(l_{0}, x=y\right), \varnothing,\left\langle\left(l_{1}, x \geqslant 0 \wedge y \neq a\right),\left(l_{2}, x=y=0\right)\right\rangle\right) \neq \varnothing$ if $a \neq 0$

$-T_{4}=\left(\left(l_{0}, x=y\right), \varnothing,\left\langle\left(l_{1}, x \geqslant 0 \wedge y \neq a\right),\left(l_{2}, x=y>0\right)\right\rangle\right) \neq \varnothing$ for $a \in \mathbb{R}_{\geqslant 0}$. The set of transitions $\mathbb{T}_{1}=\left\{T_{2}, T_{3}, T_{4}\right\}$ is valid if $a \neq 0$, in which case the max. probability of reaching $l_{3}$ is 0.5 , and $\mathbb{T}_{2}=\left\{T_{1}, T_{4}\right\}$, is valid if $a=0$, in which case the max. probability of reaching $l_{3}$ is 1 . If we wish to maximise this probability, the algorithm obtains the constraint $a=0$.

\section{Conclusion}

We presented a technique for PPTA which derives symbolic constraints on parameters of the model, such that the $\mathrm{max} / \mathrm{min}$ probability of reaching some set of locations is optimised. We focused on probabilistic reachability, but can easily consider more expressive target sets that refer to locations and clocks by syntactically modifying the model as in [18]. Computing expected time properties using game abstractions is still open for PTA. Termination of our algorithm depends on whether the forward reachability exploration terminates. Unlike for TA/PTA, where the extrapolation operator on zones can be used, in the parametric case we need to impose certain restrictions to ensure termination. One possibility is 
to restrict the parameter domain to bounded integers as in [11]. We are currently implementing the algorithm in PRISM.

Acknowledgments This research is supported by ERC AdG VERIWARE.

\section{References}

1. R. Alur and D. L. Dill. A theory of timed automata. TCS, 126:183-235, 1994.

2. R. Alur, T. A. Henzinger, and M. Y. Vardi. Parametric real-time reasoning. In STOC'93, pages 592-601. ACM Press, 1993.

3. É. André, L. Fribourg, and J. Sproston. An extension of the inverse method to probabilistic timed automata. FMSD, 42:119-145, 2013.

4. N. Chamseddine, M. Duflot, L. Fribourg, C. Picaronny, and J. Sproston. Computing expected absorption times for parametric determinate probabilistic timed automata. In QEST'08, pages 254-263. IEEE CS Press, 2008.

5. A. Condon. The complexity of stochastic games. Information and Computation, 96:203-224, 1992.

6. M. Diciolla, C. H. P. Kim, M. Kwiatkowska, and A. Mereacre. Synthesising optimal timing delays for timed i/o automata. In EMSOFT'14. ACM, 2014.

7. E. M. Hahn, H. Hermanns, and L. Zhang. Probabilistic reachability for parametric markov models. In SPIN, pages 88-106, 2009.

8. T. Han, J.-P. Katoen, and A. Mereacre. Approximate parameter synthesis for probabilistic time-bounded reachability. In RTSS, pages 173-182. IEEE Computer Society, 2008.

9. T. Hune, J. Romijn, M. Stoelinga, and F. W. Vaandrager. Linear parametric model checking of timed automata. Journal of Logic and Algebraic Programming, 52-53:183-220, 2002.

10. A. Jovanović and M. Kwiatkowska. Parameter synthesis for probabilistic timed automata using stochastic game abstractions. Technical Report CS-RR-14-06, Oxford University, June 2014.

11. A. Jovanović, D. Lime, and O. H. Roux. Integer parameter synthesis for timed automata. In TACAS 2013, volume 7795 of $L N C S$, pages 401-415. Springer, 2013.

12. A. Jovanović, D. Lime, and O. H. Roux. Synthesis of bounded integer parameters for parametric timed reachability games. In ATVA 2013, volume 8172 of LNCS. Springer, 2013.

13. M. Kattenbelt, M. Kwiatkowska, G. Norman, and D. Parker. A gamebased abstraction-refinement framework for Markov decision processes. FMSD, 36(3):246-280, 2010.

14. M. Kwiatkowska, G. Norman, and D. Parker. Game-based abstraction for Markov decision processes. In QEST'06, pages 157-166. IEEE CS Press, 2006.

15. M. Kwiatkowska, G. Norman, and D. Parker. Stochastic games for verification of probabilistic timed automata. In FORMATS'09, volume 5813 of LNCS, pages 212-227. Springer, 2009.

16. M. Kwiatkowska, G. Norman, and D. Parker. PRISM 4.0: Verification of probabilistic real-time systems. In CAV'11, volume 6806 of $L N C S$, pages 585-591. Springer, 2011.

17. M. Kwiatkowska, G. Norman, D. Parker, and J. Sproston. Performance analysis of probabilistic timed automata using digital clocks. FMSD, 29:33-78, 2006.

18. M. Kwiatkowska, G. Norman, R. Segala, and J. Sproston. Automatic verification of real-time systems with discrete probability distributions. TCS, 282:101-150, 2002. 